\title{
Unterstützung Kreativer Arbeit mit Crowdsourcing
}

\author{
Jonas Oppenländer \\ jonas.oppenlaender@oulu.fi \\ University of Oulu \\ Oulu, Finnland
}

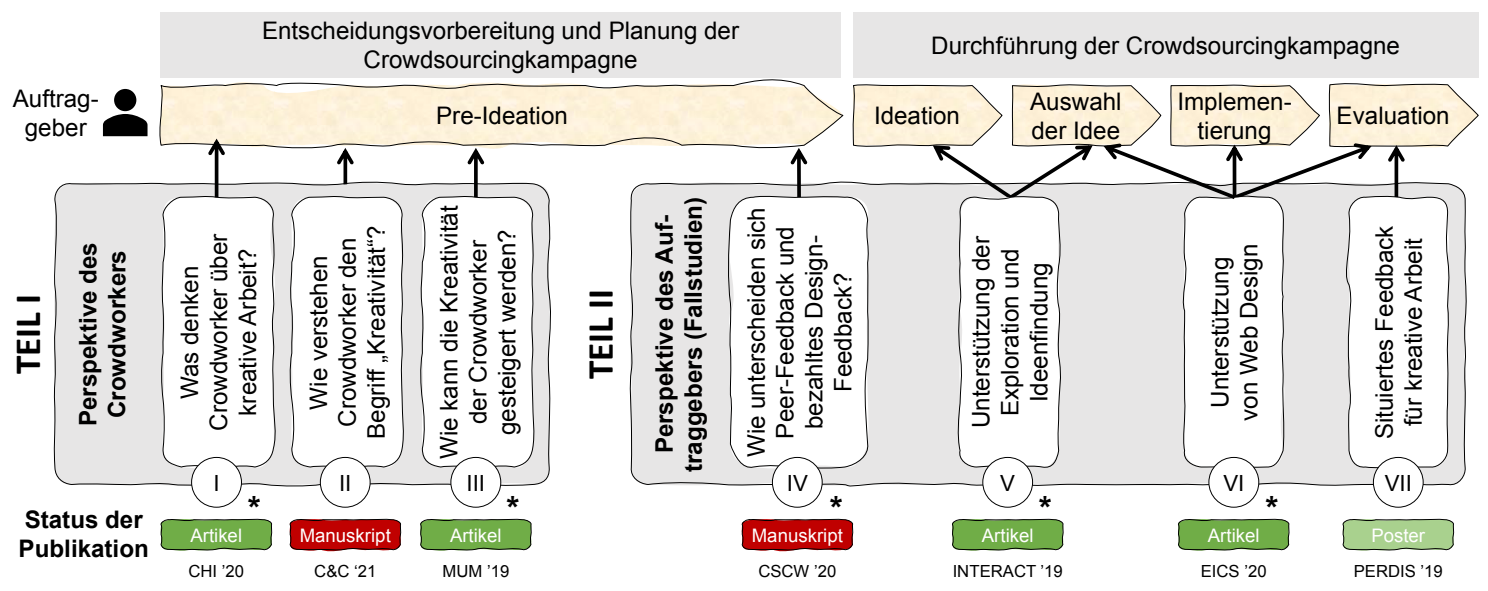

Figure 1: Übersicht über das Forschungsprojekt. Mit Asterisk markierte Arbeiten sind für die Aufnahme in die kumulative Dissertation vorgesehen.

\begin{abstract}
Diese Dissertation untersucht kreative Arbeit auf Crowdsourcingplattformen aus zwei unterschiedlichen Sichtweisen. Aus der neuartigen Perspektive des Crowdworkers wird die gefühlte Erfahrung und Einstellung zu kreativer Arbeit untersucht. Aus der Perspektive des Auftraggebers werden Themen untersucht, die der Planung und dem Design einer Crowdsourcingkampagne dienen.
\end{abstract}

\section{CCS CONCEPTS}

- Human-centered computing $\rightarrow$ Interactive systems and tools; ・ Information systems $\rightarrow$ Crowdsourcing; Decision support systems.

\section{EINFÜHRUNG UND MOTIVATION}

Das rasante Entwicklungstempo im Bereich der Technologien der künstlicher Intelligenz (KI) wird unsere Arbeitsrealität verändern. Die am Arbeitsmarkt nachgefragten Skills werden sich verschieben [7], und das Modell des "Knowledge Workers" könnte sich in Zukunft zugunsten eines "Creative Knowledge

MuC '20, 6.-9. September, 2020, Magdeburg, Deutschland

(c) 2020 Copyright held by the owner/author(s).

This is the author's version of the work. It is posted here for your personal use. Not for redistribution. The definitive Version of Record was published in $\mathrm{MuC}$ '20: Doktorandenseminar, 6.-9. September, 2020, Magdeburg, Deutschland.
Workers" [6] entwickeln. Gerade Kreativität wird in Studien zum zukünftigen Arbeitsmarkt oft unter den top-gefragten Skills gelistet (z.B. [7]). Es lohnt sich deshalb bereits heute über den Platz des Menschen in zukünftigen Wertschöpfungsketten nachzudenken.

Dieses Forschungsprojekt untersucht unter dem Motto Crowd-powered Creativity Support, wie Menschen andere Menschen mittels Crowdsourcing in komplexer kreativer Arbeit unterstützen können. Das Thema ist nicht neu [8, 24, 25], und in der Praxis wurde Crowdsourcing bereits seit über einer Dekade in vielfältigen Bereichen erforscht, erprobt, und eingesetzt [11]. In der Literatur findet sich deshalb eine große Anzahl an Arbeiten, welche sich mit der Kostenoptimierung, Arbeitsqualität, und Erprobung neuartiger Workflows befassen. Zahllose Studien über oder mit Kreativität als Bestandteil wurden auf Crowdsourcingplattformen, wie zum Beispiel Amazon Mechanical Turk, durchgeführt [9].

Jedoch waren diese Plattformen ursprünglich gar nicht für kreative Arbeit vorgesehen. Die Arbeit auf Crowdsourcingplattformen ist oft kleinteilig, monoton, und von Natur aus wenig kreativitätsfördernd. Im Kontext dieser Plattformen untersucht dieses Forschungsprojekt die erlebte Erfahrung von kreativer Arbeit aus der Sicht des Crowdworkers. Ausserdem wird in in mehreren Fallstudien untersucht, wie der Auftraggeber in seiner eigenen komplexen kreativen Arbeit unterstützt werden kann. 
Table 1: Entscheidungskriterien für das Outsourcen kreativer Arbeit (Sichtweise des Auftraggebers)

\begin{tabular}{lc}
\hline Kriterium & Artikel \\
\hline Entscheidungsvorbereitung und Planung der Crowdsourcingkampagne & \\
\hline Auswahl einer geeigneten Crowdsourcingplattform & I, II, IV, VII \\
Taskdesign und -optimierung & I, II, III, VI, VII \\
Qualität und Validität der Arbeitsergebnisse & I, II, III, IV \\
Design und Implementierung der Crowdsourcingkampagne & I, II, III, IV, V, VI, VII \\
\hline Durchführung der Crowdsourcingkampagne & V, VI \\
\hline Unterstützung der Ideenfindung & VI \\
Unterstützung der Problemidentifikation & V, VI, VII \\
Unterstützung der Exploration und Ideenauswahl & V, VI \\
Unterstützung der Implementierung & VI, VII \\
Unterstützung der Evaluation & \\
\hline
\end{tabular}

\section{HINTERGRUND}

\section{Scope in Bezug auf Crowdsourcingplattformen}

Unter Crowdsourcing wird in dieser Arbeit eine Praktik verstanden, in welcher Arbeitsaufträge (Tasks) auf einer webbasierten Plattform von einem Auftraggeber veröffentlicht werden und von einem Crowdworker gegen ein geringes Entgelt bearbeitet werden [11]. Nicht im Scope dieser Arbeit sind Crowdfunding, Citizen Science, und andere Plattformen, die unter den Begriff Crowdsourcing gefasst werden können.

Dieses Projekt befasst sich mit Crowdsourcingplattformen, in welchen Kreativität die Ausnahme, nicht die Regel ist. Darunter fallen, zum Beispiel, Plattformen wie Amazon Mechanical Turk (www.mturk.com) und Prolific (www.prolific.co). Diese Plattformen wurden zwar nicht für kreative Arbeit geschaffen, werden in der Praxis jedoch regelmässig dafür genutzt. In den zwei folgenden Abschnitten wird das in dieser Dissertation verwendete Begriffsverständnis von Kreativität und kreativer Arbeit erläutert.

\section{Scope in Bezug auf Kreativität}

Kreativität ist ein schwer definierbarer Begriff und es findet sich gerade in der Literatur zur Mensch-Computer-Interaktion (HCI) keine einheitliche Definition [8, 9]. Eine in der Literatur jedoch weit verbreitete Betrachtungsweise ist, dass kreative Arbeitsergebnisse sowohl ein Novum darstellen als auch einen Wert für eine bestimmte Benutzergruppe schaffen müssen, um als kreativ zu gelten [20, 23].

Diese Arbeit betrachtet Kreativität im Kontext von universellen Crowdsourcingplattformen auf denen eine große Vielfalt von kreativen Arbeiten beauftragt werden kann und auch wird. Die Arbeitsergebnisse werden dabei aus Sicht des Auftraggebers bewertet. Die Arbeiter auf diesen Plattformen sind meist keine Experten, wobei jedoch sicher viele Crowdworker professionelle Fähigkeiten in der Bearbeitung von kreativen Tasks haben oder durch die Plattform erworben haben [18]. Aufgrund der obigen Ausführungen liegt das Verständnis von Kreativität in dieser Dissertation deshalb an der Schnittstelle zwischen Pro-C (professioneller kreativer Dienstleistung) und little-c (Kreativität auf persönlicher Ebene, die jedoch auch einen Wert für andere Personen hat) [12].

\section{Kreative Arbeit auf Crowdsourcingplattformen}

Aus der Sichtweise des Crowdworkers kann kreative Arbeit auf Crowdsourcingplattformen drei grundlegende Formen annehmen:

(1) Kreative Tasks: Hier wird der Crowdworker im Sinne des Open Innovation-Modells gebeten, zur Ideenfinding oder Problemlösung beizutragen. Die Form des Arbeitsauftrags ist dabei variabel, und kann z.B. das Anfertigen einer Zeichnung, den Entwurf eines Werbeslogans, oder das Schreiben einer Kurzgeschichte umfassen.

(2) Kreativitätstests und Studien: Hier wird der Crowdworker gebeten, an online Studien teilzunehmen, welche entweder zum Ziel haben, die Kreativität des Crowdworkers direkt zu messen, oder Kreativität als Variable im Experimentdesign beinhalten.

(3) Kreativitätsbeurteilung: Hier wird der Crowdworker gebeten, die Kreativität eines Arbeitsergebnisses zu bewerten. Arbeitsaufträge dieser Art werden häufig zur Qualitätskontrolle (Peer-Review) der Arbeitsergebnisse anderer Crowdworker eingesetzt.

Diese Dissertation konzentriert sich auf Kreative Tasks und Kreativitätstests als zwei Teilbereiche kreativer Arbeit auf Crowdsourcingplattformen. Aus Sicht des Crowdworkers können diese Arbeitsaufträge in ihrer erlebten Erfahrung sehr ähnlich sein. In beiden Fällen müssen die Arbeitsergebnisse von Experten beurteilt werden, und es werden oft dieselben 
Unterstützung Kreativer Arbeit mit Crowdsourcing

Bewertungskriterien (z.B. Novelty, Usefulness, Fluency, und Flexibility [10]) eingesetzt.

\section{Übersicht der Arbeit}

Das Forschungsprojekt ist in zwei Teile gegliedert (siehe Abbildung 1). Der erste Teil (Artikel I-III) befasst sich mit der Perspektive des Crowdworkers. Aus Sicht des Auftraggebers untersucht der zweite Teil (Artikel IV-VII) in vier Fallstudien die gefühlte Erfahrung von Crowd-Feedback für den Auftraggeber (Artikel IV), und wie der Auftraggeber in komplexer kreativer Arbeit unterstützt werden kann (Artikel V-VII).

Sowohl Teil I als auch Teil II tragen Erkenntnisse bei, welche als Entscheidungsgrundlage für den Auftraggeber bei der Planung einer kreativen Crowdsourcingkampagne dienen können. Fünf Artikel sind für die Aufnahme in die kumulative Dissertation vorgesehen (siehe Abbildung 1). Im Folgenden werden die einzelnen Artikel kurz vorgestellt.

\section{TEIL 1: PERSPEKTIVE DES CROWDWORKERS}

\section{Was denken Crowdworker über kreative Arbeit? (I)}

Problemstellung. Motivation ist ein Faktor, welcher die Kreativität eines Arbeiters entscheidend beeinflussen kann [3]. Gerade auf Crowdsourcingplattformen, auf welchen kreative Arbeitsaufträge die Ausnahme sind, könnte die Einstellung eines Crowdworkers zu kreativer Arbeit ein Faktor sein, der die Qualität der Arbeitsergebnisse entscheidend beeinflusst [5].

Lösungsweg. Eine Umfrage auf zwei unterschiedlichen Crowdsourcingplattformen (Amazon Mechanical Turk und Prolific) mit insgesamt 215 Teilnehmern lieferte komplementäre Erkenntnisse über die Arbeitspräferenzen und Einstellung der Crowdworker zu kreativer Arbeit. Die Daten wurden mittels einer Analyse mit gemischten Methoden (jedoch mit Schwerpunkt auf qualitative Analyse) ausgewertet.

Ergebnisse. Aus der qualitativen Analyse [18] entwickelten sich fünf unterschiedliche Arbeiterprofile, mit unterschiedlicher Motivation und unterschiedlichen Präferenzen für kreative Arbeit (Professional, Casual, Pragmatic, Novelty Seeker, und Self-Developer). Professionelle Crowdworker zeigten eine Abneigung gegen kreative Arbeit, da der Arbeitserfolg hier schwer messbar und der Willkür des Auftraggebers ausgeliefert ist. Die meisten der professionellen Crowdworker gaben außerdem an, dass sie kreative Kollaboration und Experimente mit mehreren Teilnehmern eher meiden, da die Arbeit mit mehreren Personen mit technischen Problemen, Unterbrechungen und Wartezeiten verbunden sein kann.

Desweiteren fand sich, dass ein großer Anteil der Crowdworker (etwa 30\% der Personen, welche auf diese Frage antworteten) bereits mindestens einmal an standardisierten Kreativitätstests teilgenommen hatte. Insbesondere Guilfords Alternative Uses Test war unter diesen Arbeitern bekannt.
MuC '20, 6.-9. September, 2020, Magdeburg, Deutschland

Es zeigten sich deutliche Unterschiede zwischen den zwei Plattformen in Bezug auf kreative Arbeit. Auf Prolific finden sich mehr Gelegenheitsarbeiter, die gegenüber kreativen Studien und Experimenten mehr aufgeschlossen sind.

\section{Wie verstehen Crowdworker “Kreativität”? (II)}

Problemstellung. Im Bereich der Mensch-Computer-Interaktion werden Crowdworker oft gebeten, die Kreativität von Arbeitsergebnissen zu bewerten [9]. Was verstehen Crowdworker jedoch unter Kreativität? Die Suche nach einer Antwort auf diese Frage hat eine lange Tradition [1,22]. Manche Forscher (z.B. Amabile et al. [2, 4]) argumentieren, dass die Frage nach der Definition des Konstrukts Kreativität keine Rolle spielt, so lange eine Person ein in sich konsistentes Verständnis von Kreativität besitzt. Viele Studien geben jedoch bestimmte Bewertungskriterien vor (z.B. "Novelty" und "Usefulness" [23]). Diese fremddefinierten Kriterien werden eventuell von den Crowdworkern nicht oder falsch verstanden. Eine Erklärung dieser Kriterien in der eigenen Ausdrucksweise der Crowdworker könnte es den Crowdworkern erleichtern, valide Arbeitsergebnisse zu liefern.

Geplanter Lösungsweg. In einer großangelegten Crowdsourcingkampagne wird das Begriffsverständnis der Crowdworker erhoben und mit den gängigen Definitionen der Literatur abgeglichen. Zum Einsatz kommen dabei Techniken der Verarbeitung Natürlicher Sprache (NLP) sowie Machine Learning.

\section{TEIL 2: PERSPEKTIVE DES AUFTRAGGEBERS}

Steigerung der Kreativität des Crowdworkers (III)

Problemstellung. Crowdworker sind eventuell nicht in der richtigen Stimmung [21], um kreative Arbeit zu leisten, da sie eher monotone und repetitive Arbeitsaufträge gewohnt sind und zum Teil auch bevorzugen [18]. In die Rolle einer anderen Person zu schlüpfen könnte in diesem Fall zu kreativeren Arbeitsergebnissen führen [13, 14, 26].

Lösungsweg. In zwei komplementären Studien [15] wurde der Einfluss von Rollen (affektives Priming) auf die Kreativität der Arbeitsergebnisse mit gemischten Methoden untersucht.

Ergebnisse. Es konnte kein signifikanter Zusammenhang zwischen dem Annehmen einer Rolle und der Kreativität der Arbeitsergebnisse festgestellt werden. Rollen eignen sich jedoch sehr wohl für den Moment, in dem einer Person die Ideen ausgehen. Aus den Studienergebnissen leiteten sich eine Reihe von Designvorschlägen für das Design von Arbeitsaufträgen auf Crowdsourcingplattformen ab.

\section{Unterschied zwischen Peer-Feedback und bezahltem Feedback von Crowdworkern (IV)}

Problemstellung. Eine wichtige Entscheidung, welche bei der Planung einer Crowdsourcingkampagne getroffen werden 
muss, ist die Auswahl der richtigen Plattform. In dieser Arbeit werden die Unterschiede zwischen Peer-Feedback und Feedback von Crowdworkern im Kontext eines Designkurses untersucht.

Geplanter Lösungsweg. In einem Within-Subject-Experiment erhielten kleine Gruppen von insgesamt 106 Bachelorstudenten und -studentinnen formatives Feedback für einen entwickelten Anwendungsprototypen. Jede Gruppe erhielt Feedback sowohl von ihren Klassenkameraden als auch Crowdworkern. Die abschliessende Befragung der Studenten erfolgte mit einem online Fragebogen, der mit gemischten Methoden ausgewertet wird. Die Analyse der Antworten wird sich auf die gefühlte Erfahrung von Crowdsourcing-gestützem Feedback für kreative Arbeit konzentrieren.

\section{TEIL 3: FALLSTUDIEN}

In vier Fallstudien wird untersucht, wie komplexe kreative Arbeit in der Praxis mit Crowdsourcing unterstützt werden kann. Die Fallstudien werden dabei in einen generischen kreativen Prozess des Auftraggebers eingeordnet (siehe Abbildung 1 und Tabelle 1): Ideengenerierung, Ideenauswahl, Implementierung, und Evaluation [27], wobei der Prozess in der Praxis auch iterativ sein kann. Hauptaugenmerk dieser Fallstudien ist die Entwicklung von Benutzeroberflächen, welche die Exploration und Auswahl von Ideen (Artikel V), sowie die Analyse, Evaluation, und Aggregation von Ideenvorschlägen (Artikel VI) unterstützen. Ausserdem werden unterschiedliche Feedbackmechanismen miteinander verglichen (Artikel VII).

\section{Unterstützung der Ideenfindung, Exploration und IdeenauswahI (V)}

Problemstellung. Mit Crowdsourcing kann eine Vielzahl von Ideen erhoben werden, welche anschließend ausgewertet werden müssen. Die Auswahl erfolgt aufgrund bestimmter Kriterien, welche sich jedoch im Nachhinein auch ändern können.

Lösungsweg. Ein web-basiertes Interface [17] wurde entwickelt, mit dem Benutzer in der post-hoc Exploration und Auswahl von Ideenvorschlägen von Crowdworkern unterstützt werden. Ideen werden dabei aufgrund ihrer Übereinstimmung mit mehreren Suchkriterien sortiert. In einer Within-SubjectBenutzerstudie $(N=24)$ mit anschliessenden semi-strukturierten Interviews wurde das Interface mit einer Suchmaschine verglichen.

Ergebnisse. Das Interface unterstützt den Benutzer in der Exploration von Ideen aus unterschiedlichen Perspektiven, sowie in der Auswahl von Ideen, welche simultan mehrere Suchkriterien erfüllen. Die Studie fand unterschiedliche Suchund Explorationsstrategien.

\section{Unterstützung der IdeenauswahI, Implementierung und Evaluation (VI)}

Problemstellung. Webdesign ist eine komplexe kreative Tätigkeit, bei der viele Entscheidungen von einem Webdesigner für eine große Anzahl von Webseitenbesuchern getroffen werden. Das Design der Benutzeroberfläche unter Berücksichtigung aller Benutzeranforderungen ist oft nicht möglich. Die Benutzer der Webseite könnten hier einen wertvollen Beitrag leisten. Dabei stellen sich zwei Probleme. Erstens sind die Benutzer oft keine Designer, und die Unterstützung des Designers könnte den Benutzern schwer fallen. Zweitens stellt sich dem Webdesigner die Herausforderung, die eventuell große Anzahl von Benutzervorschlägen auszuwerten.

Lösungsweg. Mittels eines entwickelten Tools, CrowdUI [19], können Besucher einer Webseite dem Designer visuelle Designvorschläge machen. Die Webseitenbesucher können dabei Elemente der Webseite verschieben, verändern oder löschen. Die Vorschläge werden von CrowdUI für den Webdesigner aggregiert in einer Heatmap. Das Tool wurde evaluiert in einer Benutzerstudie mit 45 Teilnehmern und 60 Crowdworkern mit Erfahrung in Webentwicklung.

Ergebnisse. Webseitenbesucher waren in der Lage, mit geringem Training sinnvolle Designvorschläge zu machen. Die einzelnen Designvorschläge waren für Webentwicklung zwar schwer verständlich, jedoch zeigte sich die Stärke des Tools in der Aggregation der Designvorschläge.

\section{Unterstützung der Evaluation (VII)}

Problemstellung. Web-basiertes Feedback für kreative Werke kann auf unterschiedliche Weise gegeben werden, und jede erfordert eine andere Art der Auswertung des Feedbacks.

Lösungsweg. Ein Tablet-basierter Prototyp [16] wurde entwickelt, mit dem Feedback für ein digitales Artefakt gegeben werden kann. Feedback wird dabei in unterschiedlichen Formen gegeben (Multiple-Choice, Text, Sprache, Video, etc.). Der Prototyp wurde in einer vorläufigen Benutzerstudie mit 12 Teilnehmern und zwei potentiellen Auftraggebern mittels Observation und abschließenden Interviews evaluiert.

Ergebnisse. Es fanden sich Abweichungen zwischen dem Nutzen des Systems für Auftraggeber und den Wünschen der Benutzer (Feedbackgeber). Insbesondere zeigte sich, dass DesignFeedback aggregiert werden muss, um den Auftraggeber nicht zu überfordern.

\section{FAZIT}

Dieses Arbeit liefert wichtige Denkanstöße und Designvorschläge für Forscher und Praktiker mit dem Ziel, Crowdpowered Creativity Support im Bereich der Mensch-ComputerInteraktion zu etablieren. 


\section{Unterstützung Kreativer Arbeit mit Crowdsourcing}

\section{REFERENCES}

[1] Robert S. Albert and Mark A. Runco. 1999. A History of Research on Creativity. In Handbook of Creativity, Robert J. Sternberg (Ed.). Cambridge University Press, 16-31.

[2] Teresa M. Amabile. 1982. Social Psychology of Creativity: A Consensual Assessment Technique. Journal of Personality and Social Psychology 43, 5 (1982), 997-1013. https://doi.org/10.1037/0022-3514.43.5.997

[3] Teresa M. Amabile. 1983. The Social Psychology of Creativity: A Componential Conceptualization. Fournal of Personality and Social Psychology 45, 2 (1983), 357-376. https://doi.org/10.1037/0022-3514.45.2.357

[4] Teresa M. Amabile, Phyllis Goldfarb, and Shereen C. Brackfleld. 1990. Social Influences on Creativity: Evaluation, Coaction, and Surveillance. Creativity Research fournal 3, 1 (1990), 6-21. https://doi.org/10.1080/10400419009534330

[5] Dana Chandler and Adam Kapelner. 2013. Breaking Monotony with Meaning: Motivation in Crowdsourcing Markets. fournal of Economic Behavior \& Organization 90 (2013), 123-133. https://doi.org/10.1016/j.jebo.2013.03.003

[6] Ernest Edmonds and Linda Candy. 2002. Creativity, Art Practice, and Knowledge. Commun. ACM 45, 10 (Oct. 2002), 91-95. https://doi.org/10.1145/570907.570939

[7] World Economic Forum. 2016. The Future of Jobs. Employment, Skills and Workforce Strategy for the Fourth Industrial Revolution. Global Challenge Insight Report, Ref. 010116.

[8] Jonas Frich, Lindsay MacDonald Vermeulen, Christian Remy, Michael Mose Biskjaer, and Peter Dalsgaard. 2019. Mapping the Landscape of Creativity Support Tools in HCI. In Proceedings of the 2019 CHI Conference on Human Factors in Computing Systems (CHI '19). ACM, New York, NY, USA, Article Paper 389, 18 pages. https://doi.org/10.1145/3290605.3300619

[9] Jonas Frich, Michael Mose Biskjaer, and Peter Dalsgaard. 2018. Twenty Years of Creativity Research in Human-Computer Interaction: Current State and Future Directions. In Proceedings of the 2018 Designing Interactive Systems Conference (DIS '18). ACM, New York, NY, USA, 1235-1257. https://doi.org/10.1145/3196709.3196732

[10] J.P. Guilford. 1967. The Nature of Human Intelligence. McGraw-Hill, New York, NY, USA.

[11] Mahmood Hosseini, Alimohammad Shahri, Keith Phalp, Jacqui Taylor, and Raian Ali. 2015. Crowdsourcing: A Taxonomy and Systematic Mapping Study. Computer Science Review 17 (2015), 43-69. https://doi.org/10.1016/j.cosrev.2015.05.001

[12] James C. Kaufman and Ronald A. Beghetto. 2009. Beyond Big and Little: The Four C Model of Creativity. Review of General Psychology 13, 1 (2009), 1-12. https://doi.org/10.1037/a0013688

[13] Sheena Lewis, Mira Dontcheva, and Elizabeth Gerber. 2011. Affective Computational Priming and Creativity. In Proceedings of the SIGCHI Conference on Human Factors in Computing Systems (CHI '11). ACM, New York, NY, USA, 735-744. https://doi.org/10.1145/1978942.1979048

[14] Robert R. Morris, Mira Dontcheva, and Elizabeth M. Gerber. 2012 Priming for Better Performance in Microtask Crowdsourcing Environments. IEEE Internet Computing 16, 5 (Sept 2012), 13-19. https://doi.org/10.1109/MIC.2012.68

[15] Jonas Oppenlaender and Simo Hosio. 2019. Design Recommendations for Augmenting Creative Tasks with Computational Priming. In Proceedings of the 18th International Conference on Mobile and Ubiquitous
MuC '20, 6.-9. September, 2020, Magdeburg, Deutschland

Multimedia (MUM '19). ACM, New York, NY, USA, Article 35, 13 pages. https://doi.org/10.1145/3365610.3365621

[16] Jonas Oppenlaender and Simo Hosio. 2019. Towards Eliciting Feedback for Artworks on Public Displays. In Proceedings of the 2019 ACM Conference on Creativity \& Cognition (C\&C'19). ACM, New York, NY, USA, 562-569. https://doi.org/10.1145/3325480.3326583

[17] Jonas Oppenlaender, Elina Kuosmanen, Jorge Goncalves, and Simo Hosio. 2019. Search Support for Exploratory Writing. In HumanComputer Interaction - INTERACT 2019 (LNCS 11748), David Lamas, Fernando Loizides, Lennart Nacke, Helen Petrie, Marco Winckler, and Panayiotis Zaphiris (Eds.). Springer, Cham, Switzerland, 314-336. https://doi.org/10.1007/978-3-030-29387-1_18

[18] Jonas Oppenlaender, Kristy Milland, Aku Visuri, Panos Ipeirotis, and Simo Hosio. 2020. Creativity on Paid Crowdsourcing Platforms. In Proceedings of the 2020 CHI Conference on Human Factors in Computing Systems (CHI '20). ACM, New York, NY, USA, 1-14. https://doi.org/10.1145/3313831.3376677

[19] Jonas Oppenlaender, Thanassis Tiropanis, and Simo Hosio. 2020. CrowdUI: Supporting Web Design with the Crowd. Proceedings of the ACM on Human-Computer Interaction 4, EICS, Article 76 (6 2020), 28 pages. https://doi.org/10.1145/3394978

[20] Jonathan A. Plucker, Ronald A. Beghetto, and Gayle T. Dow. 2004. Why Isn't Creativity More Important to Educational Psychologists? Potentials, Pitfalls, and Future Directions in Creativity Research. Educational Psychologist 39, 2 (2004), 83-96. https://doi.org/10.1207/s15326985ep3902_1

[21] Sihang Qiu, Ujwal Gadiraju, and Alessandro Bozzon. 2020. Just the Right Mood for HIT!. In Web Engineering, Maria Bielikova, Tommi Mikkonen, and Cesare Pautasso (Eds.). Springer, Cham, Switzerland, 381-396. https://doi.org/10.1007/978-3-030-50578-3_26

[22] James Melvin Rhodes. 1956. The Dynamics of Creativity: An Interpretation of the Literature on Creativity with a Proposed Procedure for Objective Research. http:/hdl.handle.net/10150/290034 Disseration.

[23] Mark A. Runco and Garrett J. Jaeger. 2012. The Standard Definition of Creativity. Creativity Research fournal 24, 1 (2012), 92-96. https://doi.org/10.1080/10400419.2012.650092

[24] Ben Shneiderman. 2002. Creativity Support Tools. Commun. ACM 45, 10 (Oct. 2002), 116-120. https://doi.org/10.1145/570907.570945

[25] Ben Shneiderman, Gerhard Fischer, Mary Czerwinski, Mitch Resnick, Brad Myers, Linda Candy, Ernest Edmonds, Mike Eisenberg, Elisa Giaccardi, Tom Hewett, Pamela Jennings, Bill Kules, Kumiyo Nakakoji, Jay Nunamaker, Randy Pausch, Ted Selker, Elisabeth Sylvan, and Michael Terry. 2006. Creativity Support Tools: Report From a U.S. National Science Foundation Sponsored Workshop. International fournal of Human-Computer Interaction 20, 2 (2006), 61-77. https://doi.org/10.1207/s15327590ijhc2002_1

[26] Jaime Teevan and Lisa Yu. 2017. Bringing the Wisdom of the Crowd to an Individual by Having the Individual Assume Different Roles. In Proceedings of the 2017 ACM SIGCHI Conference on Creativity and Cognition (C\&C '17). ACM, New York, NY, USA, 131-135. https://doi.org/10.1145/3059454.3059467

[27] Liang Zeng, Robert W. Proctor, and Gavriel Salvendy. 2011. Can traditional divergent thinking tests be trusted in measuring and predicting real-world creativity? Creativity Research fournal 23, 1 (2011), 24-37. https://doi.org/10.1080/10400419.2011.545713 